\section{Activities of the Canadian Thoracic Society and its committees}

$\mathrm{T}$ he Canadian Thoracic Society (CTS) is a framework for supporting many activities, which are primarily (although not exclusively) embedded within its committees. The majority of CTS board members are chairs of the various standing and ad hoc CTS committees. The CTS board also has a number of members who do not sit on committees, but who act as liaisons with other organizations, associations and societies.

In the present report, you will gain a high-level view of a sampling of the major projects we are engaged in. The list is not meant to be exhaustive. Beyond these specific projects, many of the committees are also involved in activities, such as providing media spokespersons on various issues and taking part in the Canadian Respiratory Conference and CHEST scientific programs.

I am grateful to Suzanne Desmarais for collating the information in the present report.

\section{PEDIATRIC ASSEMBLY COMMITTEE}

The CTS is delighted to have welcomed the Pediatric Assembly within its ranks. The Assembly met in San Francisco (California, USA) in May 2007 and voted to incorporate into the CTS. The nature of our relationship with the Pediatric Assembly will be further defined as part of the upcoming review of the CTS rules and regulations. We look forward to working with the Assembly to advance pediatric respirology in Canada. Welcome to all pediatric respirologists!

Chair: Tom Kovesi

Key activities:

- advocate and advise on issues related to pediatric respiratory medicine and health

- bring the pediatric perspective to the CTS board, committees and activities

- spearhead the development of guidelines and educational tools in various areas of pediatric respiratory care

\section{CANADIAN RESPIRATORY GUIDELINES COMMITTEE}

Chair: Louis-Philippe Boulet

Key activities:

- standardize the process of producing and updating guidelines

- develop strategies for guideline dissemination, implementation and evaluation

\section{Les activités de la Société canadienne de thoracologie et de ses comités}

T a Société canadienne de thoracologie (SCT) est une organisaLtion qui apporte son concours à de nombreuses activités, dont la plupart relèvent de comités. La majorité des membres du conseil d'administration sont des présidents ou présidentes de comités permanents ou de comités spéciaux de la SCT. D'autres membres du conseil ne siègent pas à des comités mais agissent à titre d'agent de liaison avec des organisations, des associations ou des sociétés.

Le présent rapport donne un bon aperçu des principaux projets dans lesquels s'est engagée la Société, bien que la liste ne soit pas exhaustive. Outres ces projets, bon nombre de comités participent à d'autres activités, par exemple s'adresser aux médias sur différents sujets par la voix d'un ou d'une porte-parole ou participer à l'élaboration des programmes scientifiques du Congrès canadien sur la santé respiratoire et du congrès CHEST.

Par ailleurs, je tiens à remercier $\mathrm{M}^{\mathrm{me}}$ Suzanne Desmarais pour la collecte de renseignements nécessaires à la rédaction du rapport.

\section{COMITÉ DE LA PEDIATRIC ASSEMBLY}

C'est avec fierté que la SCT a accueilli en son sein la Pediatric Assembly. Cette dernière s'est réunie à San Francisco, en Californie, en mai 2007 et a décidé de joindre les rangs de la SCT. La nature des relations avec la Pediatric Assembly reste à définir dans le cadre du prochain examen des règlements de la SCT. Nous nous réjouissons à l'idée de travailler en collaboration avec l'Assembly en vue de faire progresser la pneumologie pédiatrique au Canada. Bienvenue à tous les pneumologues pédiatres!

Président : Tom Kovesi

Principales activités

- Faire valoir les problèmes liés à la médecine et à la santé respiratoires pédiatriques et donner des conseils en la matière

- Présenter le point de vue de la pneumologie pédiatrique au conseil d'administration et aux comités de la SCT ainsi que dans les différentes activités

- Être le fer de lance de l'élaboration de lignes directrices et d'outils de formation dans différents domaines de la pneumologie pédiatrique

\section{COMITÉ DES LIGNES DIRECTRICES CANADIENNES EN PNEUMOLOGIE}

Président : Louis-Philippe Boulet

Principales activités

- Uniformiser le processus d'élaboration et de mise à jour des lignes directrices

- Élaborer des stratégies de diffusion, de mise en œuvre et d'évaluation des lignes directrices 
- facilitate rapid translation of guidelines into improvements in respiratory health

- secure stable funding for guideline activities

- prioritize guidelines initiatives

- work with asthma, chronic obstructive pulmonary disease (COPD) and sleep apnea committees to develop workshops/sessions at the Canadian Respiratory Conference

- develop and launch a Canadian Respiratory Guidelines Web site

\section{ASTHMA COMMITTEE}

Chair: Andrew McIvor

Key activities:

- update Canadian asthma management recommendations

- develop evidence-based case studies for publication in the Canadian Medical Association Journal

- produce slide kits for continuing medical education (CME)

- develop an on-line asthma management course in collaboration with the CTS Professional Development Committee and Memorial University of Newfoundland (St John's, Newfoundland and Labrador)

\section{COPD AND REHABILITATION COMMITTEE}

Chair: Denis O'Donnell

Key activities:

- "Canadian Thoracic Society recommendations for management of chronic obstructive pulmonary disease: 2007 update" (published)

- highlights of the COPD guidelines for family physicians and 'slim jim' (published)

- COPD guidelines slide kit with case studies; PDA version of the guidelines (spring 2008)

\section{HOME VENTILATION}

Co-chairs: Douglas McKim and Jeremy Road

Key activities:

- conduct consensus recommendations at the Canadian Respiratory Conference (June 2008)

- produce guidelines on home ventilation (summer 2008)

\section{SLEEP-DISORDERED BREATHING COMMITTEE}

Chair: John Fleetham

Key activities:

- Canadian guidelines on the diagnosis and treatment of sleep apnea (published in 2006)

- conducted the Canadian Sleep Summit (April 2007)

- produced a brochure ('slim jim') aimed at family physicians to assist in diagnosis and treatment (February 2008)

- advocate for equal access to diagnosis and treatment of sleep apnea across Canada
- Faire en sorte que l'application des lignes directrices se traduise rapidement par une amélioration de la santé respiratoire

- Assurer le financement stable des activités relatives aux lignes directrices

- Établir l'ordre de priorité des initiatives liées aux lignes directrices

- Travailler en collaboration avec les comités sur l'asthme, sur la maladie pulmonaire obstructive chronique (MPOC) et sur l'apnée du sommeil afin d'organiser des ateliers ou des séances dans le cadre du Congrès canadien sur la santé respiratoire

- Élaborer et lancer un site Web sur les lignes directrices canadiennes en pneumologie

\section{Président : Andrew McIvor}

\section{COMITÉ SUR L'ASTHME}

Principales activités

- Mettre à jour les recommandations relatives à la prise en charge de l'asthme au Canada

- Élaborer des études de cas fondées sur des données probantes en vue d'une publication dans le Journal de l'Association médicale canadienne

- Produire des diaporamas en vue de la formation médicale continue

- Élaborer un cours en ligne sur la prise en charge de l'asthme, en collaboration avec le comité du perfectionnement professionnel de la SCT et l'Université Memorial de TerreNeuve, établie à St. John's (Terre-Neuve-et-Labrador)

\section{COMITÉ SUR LA MPOC ET LA RÉADAPTATION}

Président : Denis O’Donnell

Principales activités

- Préparer les « Les recommandations de la Société canadienne de thoracologie au sujet de la prise en charge de la maladie pulmonaire obstructive chronique - Mise à jour de 2007 » (publiées)

- Faire ressortir les principaux points des lignes directrices sur la MPOC à l'intention des médecins de famille et préparer un dépliant (publiés)

- Préparer un diaporama sur les lignes directrices relatives à la MPOC, accompagnées d'études de cas, et une version des lignes directrices, applicable dans un assistant numérique personnel (printemps 2008)

\section{COMITÉ SUR LA VENTILATION DOMESTIQUE}

Coprésidents : Douglas McKim et Jeremy Road

Principales activités

- Diriger les discussions sur des recommandations de consensus au cours du Congrès canadien sur la santé respiratoire (juin 2008)

- Élaborer des lignes directrices sur la ventilation domestique (été 2008) 


\section{PULMONARY VASCULAR DISEASE AD HOC COMMITTEE}

Chair: Sanjay Mehta

Key activities:

- Canadian Pulmonary Hypertension Forum (held in the spring of 2007 in Toronto, Ontario)

- operate the Canadian Pulmonary Arterial Hypertension Trials Group

- develop a Canadian pulmonary hypertension patient registry (ongoing)

- develop <www.LivingwithPH.ca>, a Web site targeting Canadian patients with pulmonary hypertension

- participate in establishing the Pulmonary Hypertension Association of Canada (a new national umbrella group to coordinate patient-related activities)

\section{Chair: Heather Ward}

\section{TUBERCULOSIS COMMITTEE}

Key activities:

- collaborate with the Canadian Lung Association and the Public Health Agency of Canada in development of the Canadian Tuberculosis Standards (6th edition)

- develop a resource for family physicians on the new Canadian Tuberculosis Standards

- develop a summary of the Advisory Committee Statement on interferon-gamma release assays for latent tuberculosis infection for publication in the Canadian Respiratory Journal

\section{CLINICAL TRIALS COMMITTEE}

The Canadian Respiratory Clinical Research Consortium, under the leadership of the CTS Clinical Trials Committee, is an umbrella organization of professional societies with a special interest in respiratory-related clinical research.

Chair: Shawn Aaron

Key activities:

- facilitate progress of ongoing clinical research projects

- communicate with the Canadian respiratory community about clinical research endeavours by holding a national conference each year

- develop a Canadian Institutes of Health Research team grant in clinical respiratory research (submitted)

- develop a new study focusing on COPD, asthma and sleep-disordered breathing (underway)

- conduct a Canadian obesity-asthma study (underway)

- Canadian Optimal Therapy of COPD Trial (published)

\section{RESEARCH COMMITTEE}

Chair: Irvin Mayers

Key activities:

- CTS Research Fellowship Competition launched (part of the Canadian Lung Association National Research Programs)

\section{COMITÉ SUR LES TROUBLES RESPIRATOIRES DU SOMMEIL}

Président : John Fleetham

Principales activités

- Élaborer des lignes directrices canadiennes sur le diagnostic et le traitement de l'apnée du sommeil (publiées en 2006)

- Diriger le Canadian Sleep Summit (avril 2007)

- Préparer un dépliant à l'intention des médecins de famille sur le diagnostic et le traitement de ces troubles (février 2008)

- Plaider en faveur d'un accès égal aux ressources pour le diagnostic et le traitement de l'apnée du sommeil, partout au Canada

\section{COMITÉ SPÉCIAL SUR LA MALADIE VASCULAIRE PULMONAIRE}

\section{Président : Sanjay Mehta}

Principales activités

- Organiser le Forum canadien sur l'hypertension pulmonaire (Toronto, printemps 2007)

- Gérer le groupe d'essais sur l'hypertension artérielle pulmonaire au Canada

- Constituer un registre des patients atteints d'hypertension artérielle pulmonaire au Canada (en cours)

- Élaborer un site Web (www.LivingwithPH.ca) à l'intention des patients atteints d'hypertension artérielle pulmonaire au Canada

- Participer à la mise sur pied de la Pulmonary Hypertension Association of Canada (nouveau groupe national de coordination des activités organisées à l'intention des patients)

\section{COMITÉ SUR LA TUBERCULOSE}

Présidente : Heather Ward

Principales activités

- Collaborer avec l'Association pulmonaire du Canada et l'Agence de la santé publique du Canada relativement à l'élaboration des Normes canadiennes pour la lutte antituberculeuse ( $6 \mathrm{e}$ édition)

- Concevoir une ressource à l'intention des médecins de famille sur les nouvelles Normes canadiennes pour la lutte antituberculeuse

- Préparer un résumé de la déclaration du comité consultatif sur les Tests de libération d'interféron-gamma pour la détection de l'infection tuberculeuse latente en vue d'une publication dans la Revue canadienne de pneumologie

\section{COMITÉ DES ESSAIS CLINIQUES}

Le Consortium canadien de recherche clinique en pneumologie, sous la direction du comité des essais cliniques de la SCT, est une organisation qui chapeaute plusieurs sociétés professionnelles portant un intérêt particulier à la recherche clinique en pneumologie.

Président : Shawn Aaron

Principales activités

- Faciliter la poursuite des projets de recherche clinique en cours

- Communiquer avec la collectivité en pneumologie au Canada sur les projets de recherche clinique par la tenue annuelle d'un congrès national 
- peer review and ranking of fellowship applications, as well as make recommendations for funding

- provide advice on research issues

- guide the development of a national respiratory research agenda

\section{PROFESSIONAL DEVELOPMENT COMMITTEE}

Chair: George Fox

Key activities:

- accredit CME/continuing professional development programs under the Royal College of Physicians and Surgeons of Canada (RCPSC) Maintenance of Certification Program (section 1)

- liaise with the RCPSC with regard to accreditation issues

- develop an on-line CME course in collaboration with the Asthma Committee and Memorial University of Newfoundland

\section{PROGRAM PLANNING SUBCOMMITTEE}

Chair: Robin McFadden

Key activities:

- plan the CTS Scientific Program for CHEST

- co-chair the Canadian Respiratory Conference Scientific Committee

- plan the annual Christie Memorial Lecture

- collaborate with the Canadian Institutes of Health Research on the Institute of Circulatory and Respiratory Health Distinguished Lecture in Respiratory Sciences

\section{ANNUAL RESIDENT/FELLOW RESEARCH COMPETITION}

Chair: Gerard Cox

Key activities:

- conduct an annual two-day competition to showcase the research of Respiratory and Allergy and Immunology residents and fellows

- foster an interest in research and provide an informal forum for the exchange of ideas among young researchers

\section{RESPIRATORY FELLOW EXAMINATION PREPARATION COURSE}

Coordinator: Jennifer Wilson

Key activities:

- conduct an annual two-day course to help respiratory residents/fellows prepare for the RCPSC examination

\section{MEMBERSHIP COMMITTEE}

Chair: Stephen Field

Membership Engagement Advisor: Shannon Walker

Key activities:

- develop strategies for membership recruitment and retention

- promote CTS membership in the respiratory community
- Constituer une subvention d'équipe des Instituts de recherche en santé du Canada pour de la recherche en pneumologie clinique (soumise)

- Élaborer une nouvelle étude sur la MPOC, l'asthme et les troubles respiratoires du sommeil (en cours)

- Mener une étude canadienne sur le lien entre l'asthme et l'obésité (en cours)

- Réaliser un essai canadien de traitement optimal de la MPOC (publié)

\section{COMITÉ DE RECHERCHE}

Président : Irvin Mayers

Principales activités

- Lancer le concours des bourses de recherche de la SCT (dans le cadre des programmes nationaux de recherche de l'Association pulmonaire du Canada)

- Effectuer de la révision par les pairs et classer les demandes de bourse par ordre de mérite, en plus de formuler des recommandations sur le financement

- Donner des conseils sur des sujets de recherche

- Guider l'élaboration d'un programme national de recherche en pneumologie

\section{COMITÉ DU PERFECTIONNEMENT PROFESSIONNEL}

Président : George Fox

Principales activités

- Approuver les programmes de perfectionnement professionnel et de formation médicale continue conformément au programme de Maintien du certificat du Collège royal des médecins et chirurgiens du Canada (partie I)

- Établir des liens avec le Collège royal relativement à des questions d'approbation

- Élaborer un cours de formation médicale continue en ligne en collaboration avec le comité sur l'asthme et l'Université Memorial de Terre-Neuve

\section{SOUS-COMITÉ DE LA PLANIFICATION DES PROGRAMMES}

Président : Robin McFadden

Principales activités

- Planifier le programme scientifique de la SCT en vue du congrès CHEST

- Coprésider le comité scientifique du Congrès canadien sur la santé respiratoire

- Planifier la conférence commémorative Christie, organisée chaque année

- Travailler en collaboration avec les Instituts de recherche en santé du Canada en vue des conférences de l'Institut de la santé circulatoire et respiratoire, prononcées par d'éminents spécialistes en pneumologie 
- provide advice on membership issues and on communicating with members

- develop a new 'conference booth', brochure and banner (completed in 2007)

\section{STANDARDS COMMITTEE}

Chair: Nigel Duguid

Key activities:

- review documents produced by the CTS or external organizations

- make recommendations to the CTS executive with regard to CTS support or endorsement of those documents (mostly guidelines or other educational documents and tools)

\section{LONG-TERM PLANNING COMMITTEE}

Chair: Gerard Cox

Key activities:

- provide advice and guidance to the CTS executive

There are lots of exciting initiatives in process! For more information regarding the various activities of the CTS (work plan, timelines, suggestions, etc) or if you would like to become directly involved, please contact me, Janet Sutherland (CTS Director) or any of the committee chairs.

We want your ideas and involvement!

Respectfully,

Robert D Levy MD FRCPC President, Canadian Thoracic Society

\section{CONCOURS ANNUEL DE RECHERCHE DES RÉSIDENTS ET DES BOURSIERS}

Président : Gerard Cox

Principales activités

- Tenir un concours annuel de deux jours pour présenter les travaux de recherche des résidents et des boursiers en pneumologie, en allergologie et en immunologie

- Accroître l'intérêt pour la recherche et offrir aux jeunes chercheurs un cadre libre d'échange d'idées

\section{COURS PRÉPARATOIRE À L'EXAMEN D'ASSOCIÉ EN PNEUMOLOGIE}

Coordonnatrice : Jennifer Wilson

Principales activités

- Tenir un cours annuel de deux jours pour aider les résidents et les boursiers en pneumologie à se préparer à l'examen du Collège royal

\section{COMITÉ D'ADHÉSION}

Président : Stephen Field

Conseiller en matière de mobilisation des membres : Shannon Walker

Principales activités

- Élaborer des stratégies sur l'adhésion et la conservation des membres

- Promouvoir l'adhésion à la SCT au sein de la collectivité en pneumologie

- Donner des conseils sur des questions relatives à l'effectif et sur les communications avec les membres

- Renouveler le stand, la brochure et le panneau de la Société (réalisés en 2007)

Président : Nigel Duguid

\section{COMITÉ DES NORMES}

Principales activités

- Examiner les documents produits par la SCT ou des organisations externes

- Formuler des recommandations à la direction de la SCT sur le fait d'approuver ou d'appuyer ces documents (surtout des lignes directrices ou d'autres documents didactiques et des outils)

COMITÉ DE LA PLANIFICATION À LONG TERME

Président : Gerard Cox

Principales activités

- Conseiller la direction de la SCT en la matière et donner des indications sur l'orientation à prendre

Une foule d'activités toutes aussi intéressantes les unes que les autres s'offrent à vous. Si vous désirez obtenir des renseignements sur les différentes activités de la SCT (plan de travail, échéanciers, suggestions, etc.) ou si vous désirez apporter votre concours, veuillez communiquer avec moi-même, avec Janet Sutherland (directrice de la SCT) ou avec l'un des présidents ou présidentes de comités.

Nous comptons sur vous!

Robert D. Levy, MD, FRCPC

Président de la Société canadienne de thoracologie 


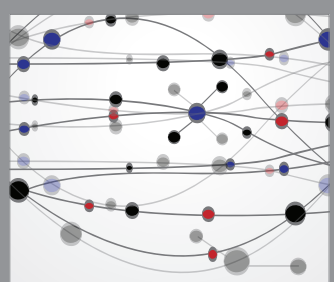

The Scientific World Journal
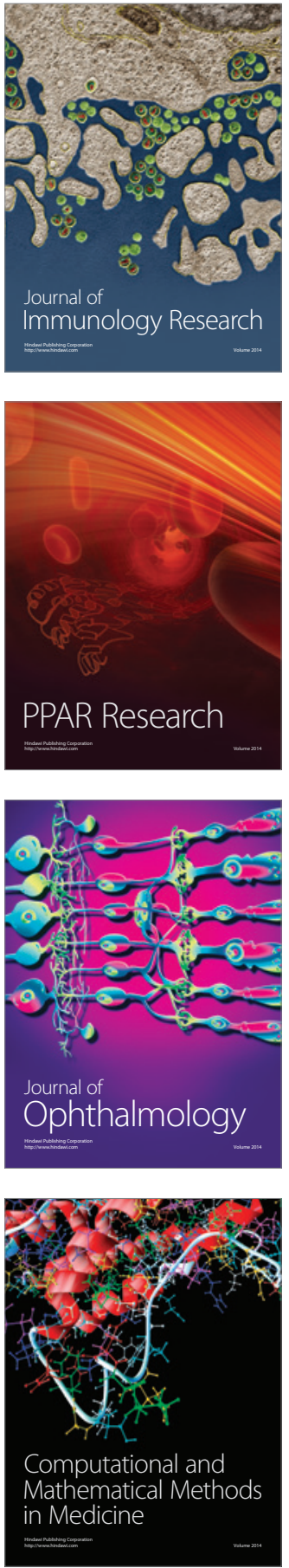

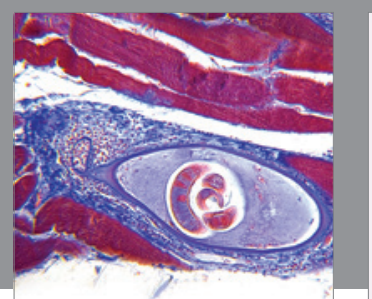

Gastroenterology Research and Practice

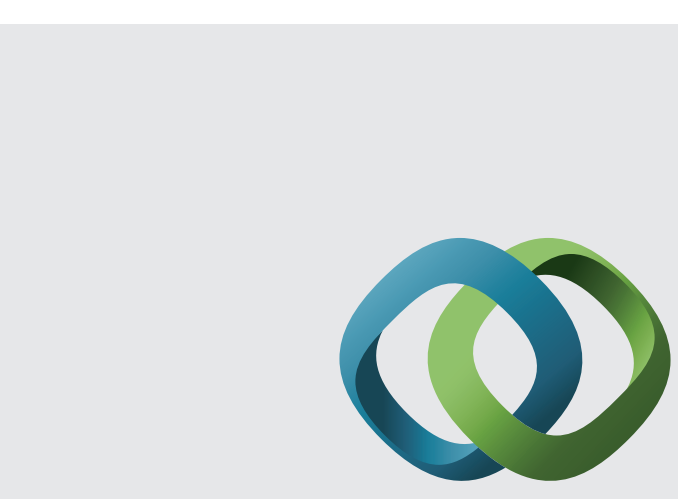

\section{Hindawi}

Submit your manuscripts at

http://www.hindawi.com
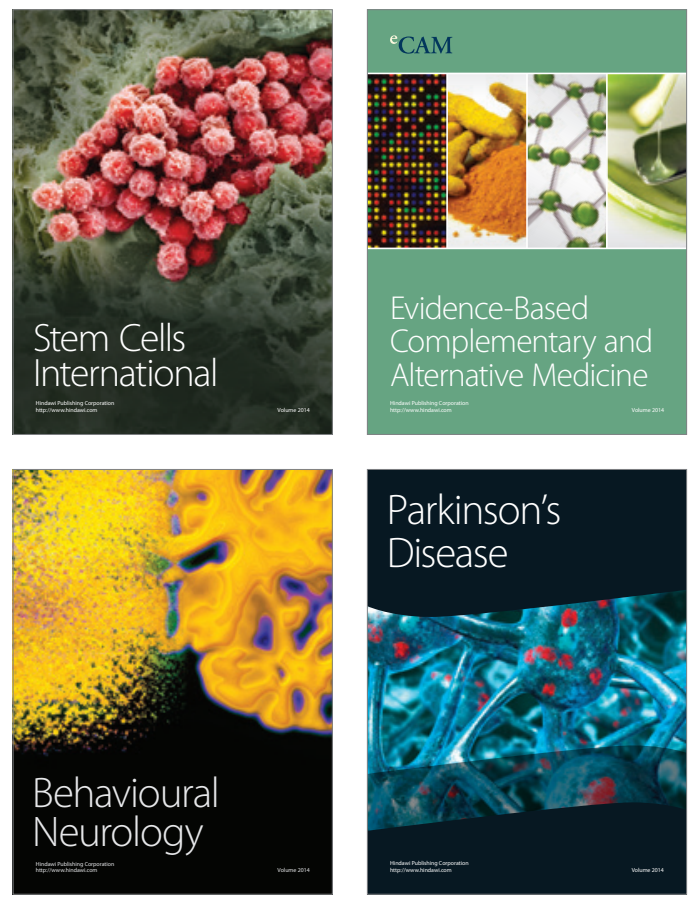
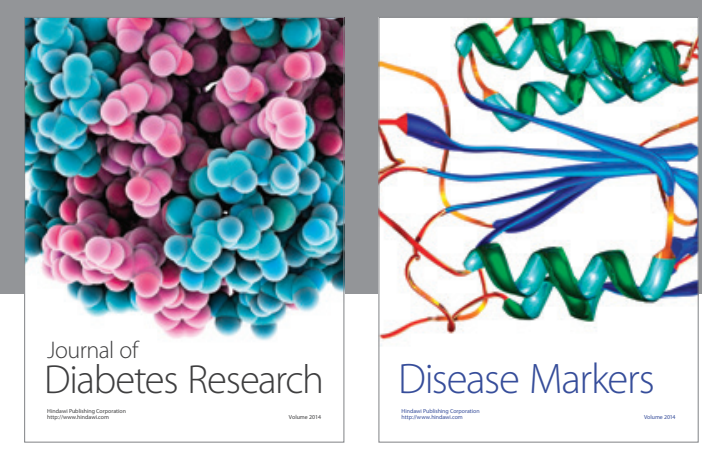

Disease Markers
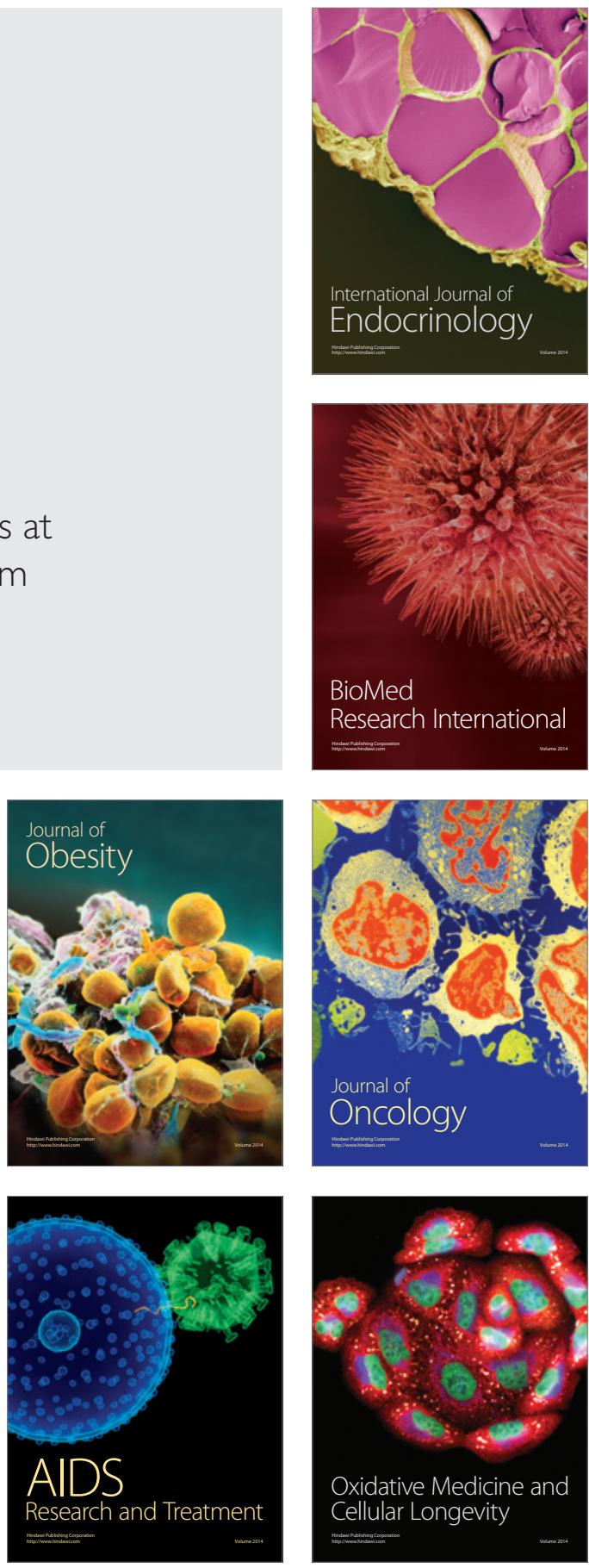